\title{
Relative Clock Synchronization in Wireless Networks
}

\author{
Iman Shames and Adrian N. Bishop
}

\begin{abstract}
This letter introduces a simple convex, constraint based, optimization protocol for the problem of relative clock synchronization in wireless (sensor) networks.
\end{abstract}

Index Terms-Clock synchronization, wireless networks.

\section{INTRODUCTION}

W IRELESS sensor networks provide a novel solution to a wide range of problems across a number of application areas. In many applications [1], [2], individual sensor nodes are often required to attach so-called "when" and "where" information to the underlying sensed data.

The problem of relative clock synchronization for wireless networks (or distributed networks more generally) is not new [1], [2] and a number of protocols exist. We consider algorithms based on the time-of-arrival (TOA) stamps measured at individual sensors from a limited number of wireless signals transmitted by certain neighbour nodes in the network. The measurements at sensor $i$ typically take the form

$$
\widehat{t}_{i j}^{\kappa}=t_{i j}^{\kappa}+e_{i}^{\kappa}=\tau_{i}^{\kappa}+\frac{r_{i j}}{v}+e_{i}^{\kappa}
$$

for a signal, indexed by $\kappa$, transmitted from sensor $j$. The inter-sensor range is $r_{i j}$ and the speed of signal propogation is $v$. Here, $e_{i}^{\kappa}$ is a zero-mean Gaussian distributed random variable with variance $\sigma_{e i}^{2}$. In general, we assume that $e_{i}^{\kappa}$ and $e_{j}^{\kappa}$ are independent. The time $\tau_{i}^{\kappa}$ is the unknown time of transmission for message $\kappa$ measured in node $i$ 's internal time frame. We assume that node $j$ transmits

$$
\widehat{\tau}_{j}^{\kappa}=\tau_{j}^{\kappa}+n_{j}^{\kappa}
$$

to node $i$ where $\tau_{j}^{\kappa}$ is the time of signal transmission as measured in node $j$ 's time frame. We assume $n_{i}^{\kappa}$ is a zeromean Gaussian distributed random variable with variance $\sigma_{n i}^{2}$.

Note that $t_{i j}^{\kappa}=\tau_{i}^{\kappa}+\frac{r_{i j}}{v}=\tau_{j}^{\kappa}+\left(\tau_{i}^{\kappa}-\tau_{j}^{\kappa}\right)+\frac{r_{i j}}{v}$ and we can now introduce the following definition.

Definition 1: The relative clock bias at $i$ is given by $\beta_{i j}=$ $\tau_{j}-\tau_{i}=-\beta_{j i}$. If node $i$ knows $\beta_{i j}$ for all nodes $j$ within node $i$ 's communication range then node $i$ is said to be synchronized. Moreover, if all nodes are synchronized then the network is said to be globally synchronized. Two nodes $i$ and $j$ within communication range are said to be adjacent.

We assume that events stamped in node $j$ 's internal clock as $\theta_{j}$ which are transmitted to an adjacent node $i$ are then transformed into node $i$ 's internal frame using $\theta_{i}=\theta_{j}-\beta_{i j}$.

Manuscript received October 12, 2009. The associate editor coordinating the review of this letter and approving it for publication was X. Zhang.

This work is supported by NICTA, which is funded by the Australian Government as represented by the Department of Broadband, Communications and the Digital Economy.

I. Shames and A. N. Bishop are with the Australian National University (ANU) and NICTA (e-mail: iman.shames@anu.edu.au).

Digital Object Identifier 10.1109/LCOMM.2010.04.092008
The simplest approach for pair-wise clock synchronization is to compute the estimate

$$
\widetilde{\beta}_{i j}=\frac{\left(\widehat{t}_{j i}^{a}-\widehat{\tau}_{i}^{a}\right)-\left(\widehat{t}_{i j}^{b}-\widehat{\tau}_{j}^{b}\right)}{2}
$$

when $i$ and $j$ are adjacent. Node $i$ broadcasts a packet $a$ to node $j$ along with $\widehat{\tau}_{i}^{a}$. Node $j$ measures $\widehat{t}_{j i}^{a}$ and subsequently returns a packet $b$ to node $i$ along with $\widehat{t}_{j i}^{a}$ and $\widehat{\tau}_{j}^{b}$. Node $i$ then measures $\widehat{t}_{i j}^{b}$ and computes $\widetilde{\beta}_{i j}$. Given multiple measurements over time then taking the average of $\widehat{\tau}_{j}^{i}$ results in the unconstrained maximum likelihood estimate (MLE) for $\widetilde{\beta}_{i j}$ [2].

Assumption 1: The clock drift is neglible over short time intervals, e.g. those considered in this work. That is, the true relative bias $\beta_{i j}=\frac{1}{2}\left[\left(t_{j i}^{a}-\tau_{i}^{a}\right)-\left(t_{i j}^{b}-\tau_{j}^{b}\right)\right]$ is independent of the packet indices $a$ and $b$.

The solution to (3) is widely used and is a variant on the remote clock reading algorithm [1]. A wide range of distributed clock synchronization prototols are based on variants of this technique [2]. A widely referenced technique known as the reference broadcast synchronization (RBS) protocol can be regarded as a simplified variant of the above approach [3] where a single node is elected as a reference transmitter (at least within defined clusters) and the time difference between time stamps receieved at adjacent nodes amounts to a bias estimate. Other techniques such as the Timing-sync Protocol for Sensor Networks (TPSN) [4], the Flooding-Time Synchronization Protocol (FTSP) [5], the Routing Integrated Time Synchronization protocol (RITS) [6] and others also use a variant of the above solution with various modifications, e.g. the broadast technique and toplogy, compensation for drift etc. For brevity, we point to the survey [2] for details.

Alternatively, a number of algorithms based on the idea of distributed consensus (developed in the control community [7]) have recently been introduced [8]-[10]. Many of these techniques have the advantage of being truly distributed. However, such protocols typically neglect the addition of noisy measurements and are often based on the notion of asymptotic convergence, i.e. they require continual execution. However, some of these techniques [11] inherently solve the problem in the face of clock drift, albeit under ideal (noiseless) sensor conditions.

\section{A. Our Contribution}

Our approach is based on the relative communication protocol and equation (3). We explicitly stated that the solution (3) is unconstrained as it naively neglects the constraints imposed on the relative clock biases by the existence of network cycles. For example, consider a simple network of three sensor nodes $\{i, j, k\}$ with a triangular network topology. Now it follows from simple algebra that

$$
\beta_{i j}+\beta_{j k}+\beta_{k i}=0
$$


and thus any solution to (3) must satisfy such a constraint. We are unaware of any similar algorithm in the literature which explicitly considers such constraints.

The main contribution of this paper is a simple centralized solution to the problem of clock synchronization for wireless sensor networks based on constraint opimization. The approach introduced in this paper explicitly considers the constraints imposed on the solution by the network topology. The algorithm we propose is robust to individual node failures, measurement noise (in every input considered) and modeling uncertainties. To the best of our knowledge no other technique exploits the inherent network cyclic constraints in the convex optimization program introduced in the next section.

\section{Constraint-Based Clock Synchronization}

We start this section by formally presenting the problem that we address in this letter.

Problem 1: Consider the network $\mathcal{N}$, with underlying graph $\mathcal{G}(\mathcal{V}, \mathcal{E})$, where $\mathcal{V}=\{i\}_{i=1}^{N}$ with $|\mathcal{V}|=N$, is its vertex set and $\mathcal{E}$ is its edge set. Moreover the undirected edge $\{i, j\} \in \mathcal{E}$ if and only if nodes $i$ and $j$ are adjacent in the network, in other words one can calculate a $\widetilde{\beta}_{i j}$ as in (3). The answers to the following questions are sought.

1) In the absence of noise, under what conditions can one find $\beta_{i j}$ for any pair of nodes $i$ and $j$ in the network?

2 ) In the presence of noise, how can one improve the calculated values of $\widetilde{\beta}_{i j}$ obtained from (3)?

It is common to say two nodes $i$ and $j$ are adjacent if they are in close physical proximity to one another, i.e. $\{i, j\} \in \mathcal{E}$ iff the range $r_{i j}$ is less than some threshold $d_{t}$. We do not restrict ourselves to this case however.

In the following theorem we answer the first question posed in Problem 1.

Theorem 1: All the relative clock biases in the network, $\beta_{i j}, \forall i, j \in \mathcal{V}$, can be calculated if and only if graph $\mathcal{G}$ is connected.

Proof: For every undirected edge $\{i, j\}$ of the graph and when there is no noise in the measurements we can calculate the relative clock bias uniquely from Equation (3). If the network is connected, then there exists a path from any node $i$ from each node $j \neq i$. It is obvious that the relative clock bias $\beta_{i j}$, is uniquely determined as the sum of the clock bias differences along the path connecting $j$ to $i$. Vice versa, assume that there exists no path from node $j$ to $i$. Therefore, $j$ and $i$ belong to distinct connected components of the network. No measurement is available about the relative clock bias of each node in the component containing $j$ with respect to any node in the component containing $i$. Therefore, it is not possible that only a single relative clock bias $\beta_{i j}$ is compatible with the measurements. This completes the proof.

Now we address the second question posed in Problem 1 by proposing a method which takes a similar approach to the one proposed in [12] to improve the relative clock biases obtained from solving (3) in the presence of noise. Note that for every cycle in the network, we can enforce a constraint along the lines of (4). If $\mathcal{G}$ is a tree, then there are no redundant cyclic constraints and we cannot reduce the effect of noise on the bias estimates beyond the solution (3).
Before continuing further, we have the following definitions. Definition 2: Define the directed edge set $\mathcal{E}_{d}$ as

$$
\left.\mathcal{E}_{d}=\{(i, j) \mid\{i, j\} \in \mathcal{E}, \& i<j)\right\},
$$

furthermore, let $\mathcal{G}_{d}$ denote the directed graph (digraph) with vertex set $\mathcal{V}_{d}$ and edge set $\mathcal{E}_{d}$.

Definition 3 (Path and cycle): Let $\mathcal{G}$ be either a directed or undirected non-empty graph. A path is a non-empty graph $P=\left(V_{P}, E_{P}\right) \subset \mathcal{G}$ of the form $V_{P}=\{i\}_{i=1}^{k}$ and $E_{p}=\left\{\left(j_{i}, j_{i+1}\right)\right\}_{i=1}^{k-1}$, where $\left\{j_{1}, \cdots, j_{k}\right\}$ is a permutation of $\{1, \cdots, k\}$. The vertices $j_{2}, \cdots, j_{k-1}$ are the inner vertices of $P$. Furthermore, every sequence of edges that form a closed path in $\mathcal{G}$ and do not visit the same node twice, except the start/end node, is called cycle and it is denoted by $\Lambda$.

The direction of a cycle is the order in which the nodes are visited. We let $L(\mathcal{G})$ denote the set of all cycles of $\mathcal{G}$, and $|\Lambda|$ denote the number of edges in the cycle $\Lambda$. It should be noted that, in a digraph $\mathcal{G}_{d}$, the cycle directions are independent of the direction of the individual edges composing the cycles.

Definition 4 (Cycle vector): For $\Lambda \in L\left(\mathcal{G}_{d}\right)$, the cycle vector is the vector $1_{\Lambda} \in\{-1,0,+1\}^{|\mathcal{E}|}, \Lambda \subset \mathbb{R}^{|\mathcal{E}|}$ whose $i$-th entry is +1 if the $i$-th edge belongs to $\Lambda$ and its orientation is consistent with the orientation of $\Lambda,-1$ if the $i$-th edge belongs to $\Lambda$ and its orientation is opposite the orientation of $\Lambda$, and is 0 otherwise.

Definition 5 (Set of cycle and fundamental cycle vectors): The set of cycle vectors is $L=\left\{1_{\Lambda}, \forall \Lambda \in L\left(\mathcal{G}_{d}\right)\right\}$. A set of fundamental cycle vectors $L_{f} \subset L$ is a subset of $L$ that constitute a base for $L$. The elements of $L_{f}$ are called fundamental cycle vectors.

Given a set of fundamental cycle vectors $L_{f}$, we let $L_{f}\left(\mathcal{G}_{d}\right)$ denote the associated fundamental cycles $L_{f}(\mathcal{G})=\{\Lambda \in$ $\left.L\left(\mathcal{G}_{d}\right) \mid 1_{\Lambda} \in L_{f}\right\}$.

Definition 6 (Cycle and fundamental cycle matrix): The cycle matrix $C$ of a directed graph $G_{d}$ is the $k \times|\mathcal{E}|$ matrix $C=\left[1_{\Lambda_{1}}, \ldots, 1_{\Lambda_{k}}\right]^{\top}$ where $k$ is the dimension of $L$. The $r \times|\mathcal{E}|$ matrix $C_{f} \subset C$, with $r=\operatorname{dim}\left(L_{f}\right)$, such that each row represents a fundamental cycle vector in $L_{f}$, is called the fundamental cycle matrix

$$
C_{f}=\left[1_{\Lambda_{1}}, \ldots, 1_{\Lambda_{r}}\right]^{\top}, \forall 1_{\Lambda_{i}} \in L_{f}
$$

Note that $C_{f}$ is not unique since it depends on the choice of the fundamental cycles vectors, and it is a full rank matrix. For more information on how to calculate fundamental sets of cycles one may refer to [13].

Let $N_{i}$ be the set of all the nodes $j$ such that $(i, j) \in \mathcal{E}_{d}$. Define $B_{i} \in \mathbb{R}^{\left|N_{i}\right|}$, as the vector obtained from stacking all the pseudo-measurements $\widetilde{\beta}_{i j}$ calculated from solving (3), $\forall j \in$ $N_{i}$. Now let $B=\left[B_{i}^{\top}, \cdots, B_{N}^{\top}\right]^{\top}$, and $\Theta$ be the vector of the to be calculated estimates, where its $k$-th entry corresponds to the estimate of the $k$-th entry of $B$.

We want to solve the estimation problem in a least square sense, hence we have

$$
\begin{array}{cl}
\underset{\Theta \in \mathbb{R}^{|\mathcal{E}|}}{\operatorname{minimize}} & \|B-\Theta\|^{2} \\
\text { subject to } & C_{f}^{\top} \Theta=0
\end{array}
$$




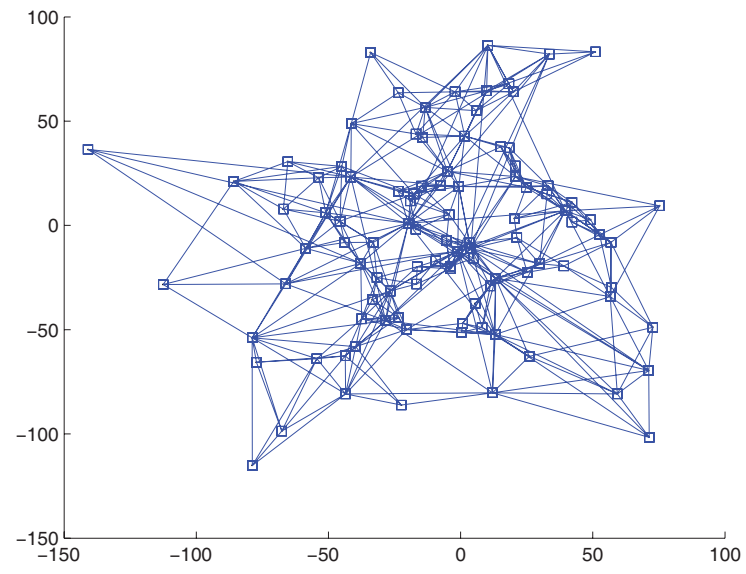

Fig. 1. An example of a network with 100 nodes that is considered here.

Moreover, we recast the constraint as $\Theta \in \operatorname{ker}\left(C_{f}^{\top}\right)$. Assume $\operatorname{ker}\left(C_{f}^{\top}\right)$ has $\epsilon_{1}, \cdots, \epsilon_{m}$ as a basis so we have

$$
\Theta=\sum_{i=1}^{m} \alpha_{i} \epsilon_{i}
$$

And the optimization problem (7) transforms into

$$
\underset{\alpha_{1}, \cdots, \alpha_{m} \in \mathbb{R}}{\operatorname{minimize}}\left\|B-\sum_{i=1}^{m} \alpha_{i} \epsilon_{i}\right\|^{2},
$$

which can be solved easily. The solution to (7) is a constrained maximum likelihood estimate under the adopted error assumptions. Furthermore, note that if the graph does not have any cycles (it is a tree), the solution to (7), will be the same as the solution obtained from solving the unconstrained equation (3) for all the edges in the network, in other words $\Theta=B$.

In the next section we present an example in which we compare the result obtained from solving the constrained optimization introduced here and the result obtained from solving the unconstrained equations of type (3).

\section{Illustrative EXAMPLE}

Consider a network of 100 nodes (An example of such network is depicted in Fig. 1). Relative clock bias estimates are calculated for different levels of measurement noise at node 1 (Node 1 is assumed to have access to all the measurements in the network.). The mean-square error in the clock bias estimate is computed over 1000 runs. The position of the nodes are randomly generated during each run according to a uniform distribution and the topology of the network is the standard proximity graph (and is thus random). The meansquare error of the estimates for both cases with considering and without considering the cycle constraints are compared with each other, as in Fig. 2.

\section{CONCLUSION}

In this letter we first formalized in which graph structure one can calculate all the relative clock biases between any pair of nodes, and then we proposed a method based on solving a convex constrained optimization problem to estimate relative clock biases in a (sensor) network. This method will in general outperform the simple unconstrained method already available

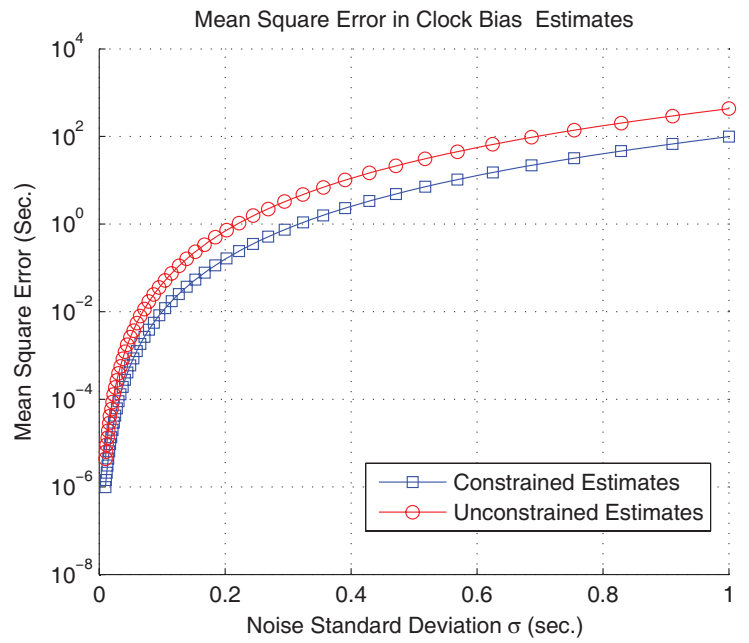

Fig. 2. The mean square error of clock bias estimates for different noise levels.

in the literature. Simulation results are presented to back this claim. Moreover, we note that it should be possible to include constraints (of the form introduced in this paper) to the (more general) algorithms presented in [3]-[6]. Finally, we highlight the fact that the method presented here does not address the problem of interest in a distributed way, and a future research direction is to develop a distributed version of this method.

\section{REFERENCES}

[1] F. Cristian, "Probabilistic clock synchronization," Distributed Computing, vol. 3, no. 3, pp. 146-158, Sep. 1989.

[2] B. Sundararaman, U. Buy, and A. D. Kshemkalyani, "Clock synchronization for wireless sensor networks: a survey," Ad Hoc Networks, vol. 3, no. 3, pp. 281-323, May 2005.

[3] J. Elson, L. Girod, and D. Estrin, "Fine-grained network time synchronization using reference broadcasts," in Proc. 5th Symposium on Operating Systems Design and Implementation, 2002.

[4] S. Ganeriwal, R. Kumar, and M. B. Srivastava, "Timing-sync protocol for sensor networks," in Proc. 1st International Conference on Embedded Networked Sensor Systems, 2003.

[5] M. Maroti, B. Kusy, G. Simon, and A. Ledeczi, "The flooding time synchronization protocol," in Proc. 2nd International Conference on Embedded Networked Sensor Systems, 2004.

[6] J. Sallai, B. Kusy, A. Ledeczi, and P. Dutta, "On the scalability of routing integrated time synchronization," in Proc. 3rd European Workshop on Wireless Sensor Networks, 2006.

[7] I. Shames, B. D. O. Anderson, and B. Fidan, "On the use of convex optimization in sensor network localization and synchronization," in Proc. 1st IFAC Workshop on Estimation and Control of Networked Systems, 2009.

[8] L. Schenato and G. Gamba, "A distributed consensus protocol for clock synchronization in wireless sensor network," in Proc. 46th IEEE Conference on Decision and Control, Dec. 2007.

[9] G. Xiong and S. Kishore, "Convergence properties of a second order consensus-based time synchronization algorithm for wireless sensor networks," in Proc. 2008 IEEE Global Communications Conference, 2008.

[10] S. Bolognani, R. Carli, and S. Zampieri, "A PI consensus controller with gossip communication for clock synchronization in wireless sensors networks," in Proc. 1st IFAC Workshop on Estimation and Control of Networked Systems, 2009.

[11] P. Sommer and R. Wattenhofer, "Gradient clock synchronization in wireless sensor networks," in Proc. 2009 International Conference on Information Processing in Sensor Networks, 2009.

[12] G. Piovan, I. Shames, B. Fidan, F. Bullo, and B. D. O. Anderson, "On frame and orientation localization for relative sensing networks," in Proc. 47th IEEE Conference on Decision and Control, pp. 2326-2331, Cancun, Mexico, Dec. 2008.

[13] J. T. Welch, "A mechanical analysis of the cyclic structure of undirected linear graphs," J. ACM, vol. 13, pp. 205-210, 1966. 